PROCEEDINGS OF THE AMERICAN MATHEMATICAL SOCIETY

Volume 135, Number 4, April 2007, Pages 1223-1232

S 0002-9939(06)08557-1

Article electronically published on September 26, 2006

\title{
A LAW OF THE ITERATED LOGARITHM FOR ARITHMETIC FUNCTIONS
}

\author{
ISTVÁN BERKES AND MICHEL WEBER
}

(Communicated by Richard C. Bradley)

\begin{abstract}
Let $X, X_{1}, X_{2}, \ldots$ be a sequence of centered iid random variables. Let $f(n)$ be a strongly additive arithmetic function such that $\sum_{p<n} \frac{f^{2}(p)}{p} \rightarrow \infty$ and put $A_{n}=\sum_{p<n} \frac{f(p)}{p}$. If $\mathbf{E} X^{2}<\infty$ and $f$ satisfies a Lindeberg-type condition, we prove the following law of the iterated logarithm:

$$
\limsup _{N \rightarrow \infty} \frac{\sum_{n=1}^{N} f(n) X_{n}}{A_{N} \sqrt{2 N \log \log N}} \stackrel{a . s .}{=}\|X\|_{2} .
$$

We also prove the validity of the corresponding weighted strong law of large numbers in $L^{1}$.
\end{abstract}

\section{MAIn RESULtS}

Let $X, X_{1}, X_{2}, \ldots$ be i.i.d. random variables with $\mathbf{E} X=0, \mathbf{E} X^{2}<\infty$. In this paper we study the weighted LIL for $\left(X_{n}\right)$, i.e. the relation

$$
\limsup _{N \rightarrow \infty} \frac{\sum_{n=1}^{N} d_{n} X_{n}}{\sqrt{2 D_{N}^{2} \log \log D_{N}^{2}}} \stackrel{\text { a.s. }}{=}\|X\|_{2},
$$

where $D_{N}^{2}=\sum_{k=1}^{N} d_{k}^{2}$. By the Hartman-Wintner law of the iterated logarithm, relation (1.1) holds if $d_{n}=1$ for all $n \geq 1$, and assuming slightly stronger moment conditions for $X,(1.1)$ will actually hold for a large class of weight sequences $\left(d_{n}\right)$. For example, a Skorohod embedding argument similar to the one used in Fisher 4 ] and Weber [11] shows that if $\mathbf{E} X^{2} \log _{+}|X|<\infty$ and

$$
D_{n} \gg n, \quad d_{n}=O\left(D_{n} n^{-\gamma}\right)
$$

for some $\gamma>0$, then (1.1) holds. Condition (1.2) covers, with $\gamma=1 / 2$, all sequences $\left(d_{n}\right)$ regularly varying with a positive exponent, and because of the arbitrary value of $\gamma$, it leaves a lot of room for irregular sequences $\left(d_{n}\right)$ as well. If we assume only $\mathbf{E} X^{2}<\infty$, the situation changes radically. In this case condition (1.2) does not suffice for the LIL (1.1), and in addition to the order of magnitude of $d_{n}$ and $D_{n}$, the distribution of the sequence $\left\{d_{n} / D_{n}, n \geq 1\right\}$ becomes crucial for the validity of (1.1). As a consequence, (1.1) fails for many irregular sequences $\left(d_{n}\right)$, and in fact,

Received by the editors May 25, 2005 and, in revised form, October 27, 2005.

2000 Mathematics Subject Classification. Primary 60F15, 11A25; Secondary 60 G50.

Key words and phrases. Iterated logarithm, strong laws of large numbers, weighted sums of iid random variables, strongly additive functions.

The first author's research was supported by the Hungarian National Foundation for Scientific Research, Grants T043037, T037886 and K61052. 
proving (1.1) for an explicitly given (irregular) sequence $\left(d_{n}\right)$ is generally a delicate problem. The situation is similar for the weighted strong law of large numbers

$$
\lim _{N \rightarrow \infty} \frac{\sum_{n=1}^{N} d_{n} X_{n}}{\sum_{n=1}^{N} d_{n}} \stackrel{\text { a.s. }}{=}\|X\|_{1}
$$

under $\mathbf{E}|X|<\infty$. Assuming slightly higher moment assumptions such as $\mathbf{E}|X| \log _{+}|X|<\infty$, one can give simple sufficient conditions for (1.3), similar to (1.2), but assuming only $X \in L_{1}$, the validity of (1.3) again becomes a delicate problem. In view of the special role irregular sequences and their distributions play in the theory, it is natural to ask if typical irregular sequences in number theory, such as additive and multiplicative arithmetic functions, can be used as coefficients in the LIL (1.1) and SLLN (1.3). The purpose of this paper is to study the case of additive functions and to prove a positive answer under natural conditions on $f$.

Let $f(n), n=1,2, \ldots$, be a strongly additive arithmetic function, namely a function $f$ satisfying

$$
\begin{aligned}
f(m n) & =f(m)+f(n), \quad(m, n)=1, \\
f\left(p^{\alpha}\right) & =f(p), \quad p \text { a prime, } \quad \alpha=2,3, \ldots
\end{aligned}
$$

It follows that $f(n)=\sum_{p} f(p) \chi(p \mid n)$, so that $f$ is completely determined by its values taken over the primes. (Here, and in the sequel, $\sum_{p}$ denotes the summation along the primes, $\chi$ denotes the indicator function and $m \mid n$ means that the integer $m$ is a divisor of the integer $n$.) A typical example is $\omega(n)$, the number of different prime factors of $n$. Put

$$
A_{n}=\sum_{p<n} \frac{f(p)}{p}, \quad B_{n}=\sum_{p<n} \frac{f^{2}(p)}{p} .
$$

By the classical central limit theorem of Erdős and Kac [3], if $|f(p)|=O(1)$ and $B_{n} \rightarrow \infty$, then we have

$$
\lim _{N \rightarrow \infty} \frac{1}{N} \#\left\{n \leq N: f(n) \leq A_{N}+x B_{N}^{1 / 2}\right\}=(2 \pi)^{-1 / 2} \int_{-\infty}^{x} e^{-u^{2} / 2} d u .
$$

The same conclusion holds for unbounded $f(p)$, provided

$$
\lim _{n \rightarrow \infty} \frac{1}{B_{n}} \sum_{\left\{p<n,|f(p)| \geq \varepsilon B_{n}^{1 / 2}\right\}} \frac{f^{2}(p)}{p}=0 \quad \text { for any } \varepsilon>0 .
$$

(See Kubilius [7, Shapiro 9.) Condition (1.7) is the analogue of the Lindeberg condition of classical probability theory and, as Kubilius [7 showed, it is also necessary for (1.6) under mild regularity conditions on the sequence $B_{n}$. Condition (1.7) is satisfied if, for example,

$$
f(p)=o\left(B_{p}^{1 / 2}\right), \quad B_{p} \rightarrow \infty
$$

The last relation is stronger than (1.7), but it is still sharp: Halberstam [5] proved that replacing the $o$ by $O$ in (1.8), the central limit theorem (1.6) becomes generally false. For additional limit theorems related to (1.6), see Kubilius [7]; for an alternative approach via the theory of mixing random variables, see Philipp [8. The purpose of this paper is to show that under condition (1.8) the sums $\sum_{n \leq N} f(n) X_{n}$ satisfy the LIL for any centered i.i.d. sequence $X_{n}$ with finite variances and the strong law of large numbers for any i.i.d. sequence $X_{n}$ with finite 
mean. This result establishes a connection between two different types of probabilistic behavior of arithmetic functions, namely the "density" type distribution result (1.6) and the almost sure asymptotic behavior of $\sum_{k=1}^{N} f(k) X_{k}$ with random $X_{k}$. As we are going to see, the key arithmetic property behind our results is a bound for the frequency of large values of $f$, and in fact a byproduct of our argument will be a large deviation result corresponding to (1.6).

Theorem 1.1. Assume that $f \geq 0$ and condition (1.8) is satisfied. Then for any sequence $X, X_{1}, X_{2}, \ldots$ of centered, independent, identically distributed, integrable random variables we have

$$
\lim _{N \rightarrow \infty} \frac{\sum_{n=1}^{N} f(n) X_{n}}{\sum_{n=1}^{N} f(n)} \stackrel{\text { a.s. }}{=} \mathbf{E} X .
$$

Theorem 1.2. Under the conditions of Theorem 1.1, for any sequence $X, X_{1}$, $X_{2}, \ldots$ of centered, independent, identically distributed, square integrable random variables we have

$$
\limsup _{N \rightarrow \infty} \frac{\sum_{n=1}^{N} f(n) X_{n}}{A_{N} \sqrt{2 N \log \log N}} \stackrel{a . s .}{=}\|X\|_{2} .
$$

In view of the law of large numbers of Jamison-Orey-Pruitt [ $[$, for the proof of Theorem 1.1 it would suffice to verify the arithmetical condition

$$
\limsup _{t \rightarrow \infty} \frac{\#\left\{N: \sum_{n=1}^{N} f(n) \leq t f(N)\right\}}{t}<\infty .
$$

Conversely, the validity of Theorem 1.1 implies (1.11). However, we could not find a direct argument for (1.11); instead we will use a suitable randomization of the function $f(n)$ in Theorems 1.1 and 1.2 and will obtain the theorems through studying the randomized function. This idea goes back to Weber [13], and our main tool will be Lemma 4.1 in [13] on the number of divisors of random sums $S_{n}$.

As we will see, (1.8) implies $B_{n}=o\left(A_{n}^{2}\right)$, and thus (1.6) describes the distribution of $f(n)$ in a short interval $\left(A_{N}(1-o(1)), A_{N}(1+o(1))\right.$ around $A_{N}$. On the other hand, relation (1.11) can be equivalently written as

$$
\#\left\{N: f(N) \geq c N A_{N}\right\} \ll 1 / c
$$

(see Lemma 2.1 below), and thus (1.11) is a large deviation result corresponding to (1.6). Our theorems show the interesting fact that such a result is valid under the same condition (1.8) required for the validity of the weak limit theorem (1.6).

Since (1.7) is the precise condition for the central limit theorem (1.6) for $f$, the question arises if (1.7) is also sufficient for the validity of Theorems 1.1 and 1.2. If $f(p)$ does not fluctuate too wildly, e.g. if

$$
\sup _{n \leq p, p^{\prime} \leq n^{2}} f(p) / f\left(p^{\prime}\right)=O(1),
$$

then this is true, as the proofs of our theorems will show. It is possible that (1.7) is sufficient without regularity conditions, but this remains open.

\section{Preparatory Lemmas}

Although Theorems 1.1 and 1.2 are formulated under condition (1.8), we will arrange the proofs to show that the Lindeberg condition (1.7) plus the mild regularity 
condition (1.12) also suffice. For this reason, in the sequel we will assume (1.7) instead of (1.8) and will indicate the places where (1.8) or the additional condition (1.12) is required.

Lemma 2.1. Under (1.7) we have

$$
\sum_{m=1}^{n} f(m) \sim n A_{n}, \quad \sum_{m=1}^{n} f^{2}(m) \sim n A_{n}^{2} .
$$

Proof. Fix $\varepsilon>0$ and let us observe that by $f \geq 0$ and the Lindeberg condition (1.7) we have for $n \geq n_{0}(\varepsilon)$

$$
\begin{aligned}
& B_{n}=\sum_{p<n} \frac{f^{2}(p)}{p}=\sum_{\left\{p<n, f(p) \geq \varepsilon B_{n}^{1 / 2}\right\}} \frac{f^{2}(p)}{p}+\sum_{\left\{p<n, f(p)<\varepsilon B_{n}^{1 / 2}\right\}} \frac{f^{2}(p)}{p} \\
& \leq \varepsilon B_{n}+\varepsilon B_{n}^{1 / 2} \sum_{p<n} \frac{f(p)}{p}=\varepsilon B_{n}+\varepsilon B_{n}^{1 / 2} A_{n}
\end{aligned}
$$

and consequently

$$
B_{n} \leq\left(\frac{\varepsilon}{1-\varepsilon}\right)^{2} A_{n}^{2}
$$

Thus we proved

$$
B_{n}=o\left(A_{n}^{2}\right)
$$

Next we observe that

$$
\sum_{p<n} \frac{1}{p}=\log \log n+c_{0}+o(1)
$$

for some constant $c_{0}$ and thus

$$
\sum_{n^{\alpha} \leq p<n} \frac{1}{p}=O(1)
$$

for any $0<\alpha<1$. Hence fixing $\varepsilon>0$ and using (1.7) we get for $n \geq n_{0}(\varepsilon)$

$$
\begin{aligned}
& B_{n}-B_{\left[n^{\alpha}\right]}=\sum_{\left\{\left[n^{\alpha}\right] \leq p<n, f(p)<\varepsilon B_{n}^{1 / 2}\right\}} \frac{f^{2}(p)}{p}+\sum_{\left\{\left[n^{\alpha}\right] \leq p<n, f(p) \geq \varepsilon B_{n}^{1 / 2}\right\}} \frac{f^{2}(p)}{p} \\
& \leq \varepsilon^{2} B_{n} \sum_{\left[n^{\alpha}\right] \leq p<n} \frac{1}{p}+\varepsilon B_{n} \leq K \varepsilon B_{n},
\end{aligned}
$$

where $K$ is a constant depending only on $\alpha$. Thus we have

$$
B_{n}-B_{\left[n^{\alpha}\right]}=o\left(B_{n}\right),
$$

and hence by a well-known result of Kubilius (see Theorem 12.1 of Elliott [2]) we get

$$
\sum_{m=1}^{n}\left(f(m)-A_{n}\right)=o\left(n B_{n}^{1 / 2}\right), \quad \sum_{m=1}^{n}\left(f(m)-A_{n}\right)^{2} \sim n B_{n},
$$

whence (2.1) follows in view of (2.2).

The next lemma is Jamison-Orey-Pruitt's characterization [6, Theorem 3] of the weighted strong law of large numbers in $L^{1}$. 
Lemma 2.2. Let $\left\{w_{k}, k \geq 1\right\}$ be a sequence of positive reals and put $W_{n}=$ $\sum_{k=1}^{n} w_{k}$. Then the relation

$$
\lim _{N \rightarrow \infty} \frac{\sum_{n=1}^{N} w_{n} X_{n}}{\sum_{n=1}^{N} w_{n}} \stackrel{\text { a.s. }}{=} \mathbf{E} X
$$

holds for all sequences $\mathcal{X}=\left\{X, X_{1}, X_{2}, \ldots\right\}$ of centered, independent, identically distributed, integrable random variables if and only if

$$
\limsup _{t \rightarrow \infty} \frac{N(t)}{t}<\infty
$$

where $N(t)=\#\left\{n: W_{n} \leq t w_{n}\right\}$.

Although (2.4) characterizes the weighted strong law of large numbers, we shall need a less elegant, but more adapted form of it. According to [6, Theorem 2, and the remark at the bottom of p. 41], the conditions

$$
\mathbf{E} X^{2} \int_{y \geq|X|} \frac{N(y)}{y^{3}} d y<\infty
$$

and $\mathbf{E}|X|<\infty$ imply (2.3), hence (2.4). Also, by the first half of the proof of Theorem 3 in [6, p. 42], relation (2.4) and $\mathbf{E}|X|<\infty$ imply (2.5), so that:

Lemma 2.3. Under the assumption $\mathbf{E}|X|<\infty$, (2.4) and (2.5) are equivalent.

This observation will be crucial in the sequel. Trying to use the original characterization (2.4) we could only establish the results in the spaces $L \log ^{\varepsilon} L, \varepsilon$ arbitrarily small but strictly positive.

The next lemma comes from Weber [13, Lemma 2.1] and estimates (2.5) in Weber 13. We also refer to Weber 14 for a precise study of the distribution of values of the divisors of i.i.d. random sums $S_{n}$, and to Weber [13 for the investigation of an extremal divisor case.

Lemma 2.4. Let $\varepsilon=\left\{\varepsilon_{i}, i \geq 1\right\}$ be a Bernoulli sequence (i.e. a sequence of i.i.d. random variables with $\mathbf{P}\left\{\varepsilon_{i}=0\right\}=\mathbf{P}\left\{\varepsilon_{i}=1\right\}=1 / 2$ ) and put $S_{n}=\varepsilon_{1}+\ldots+\varepsilon_{n}$, $n \geq 1$. There exist two absolute constants $d_{0}<\infty$ and $C<\infty$, such that for any $n \geq d \geq d_{0}$,

$$
\mathbf{E}\left\{\mathbf{1}_{d \mid S_{n}}\right\} \leq C\left(\frac{1}{n^{1 / 2}}+\frac{1}{d}\right) .
$$

For proving Theorem 1.2, we shall need a weighted version of the usual LIL. The following result is implicit in Fisher 4, see Corollary 3.4 and lines 8-11 on p. 178].

Lemma 2.5. Let $\left\{w_{k}, k \geq 1\right\}$ be a sequence of positive reals and put $T_{n}=\sum_{k=1}^{n} w_{k}^{2}$. Assume that

$$
\limsup _{t \rightarrow \infty} \frac{1}{t} \#\left\{n: T_{n} \leq t w_{n}^{2}\right\}<\infty .
$$

Then, for any sequence $\mathcal{X}=\left\{X, X_{1}, X_{2}, \ldots\right\}$ of centered, independent, identically distributed, square integrable random variables we have

$$
\limsup _{N \rightarrow \infty} \frac{\sum_{n=1}^{N} w_{n} X_{n}}{\sqrt{2 T_{N} \log \log T_{N}}} \stackrel{a . s .}{=}\|X\|_{2} .
$$


One recognizes in (2.6) condition (2.4) for the weights $w_{n}^{2}$ and in view of Lemma 2.3 it follows that under $\mathbf{E} X^{2}<\infty,(2.6)$ is equivalent to

$$
\mathbf{E} X^{4} \int_{y \geq X^{2}} \frac{\Lambda(y)}{y^{3}} d y<\infty
$$

where $\Lambda(y)=\#\left\{n: T_{n} \leq y w_{n}^{2}\right\}$.

\section{Proofs}

Proof of Theorem 1.1. Clearly $\sum_{[n / 2] \leq p<n} 1 / p \leq \sum_{[n / 2] \leq j<n} 1 / j=O(1)$, and thus using (1.7), (2.2) and the Cauchy-Schwarz inequality we get

$$
\begin{aligned}
& A_{n}-A_{[n / 2]}=\sum_{\left\{[n / 2] \leq p<n, f(p) \geq B_{n}^{1 / 2}\right\}} \frac{f(p)}{p}+\sum_{\left\{[n / 2] \leq p<n, f(p)<B_{n}^{1 / 2}\right\}} \frac{f(p)}{p} \\
& \left.\leq \sum_{\left\{p<n, f(p) \geq B_{n}^{1 / 2}\right\}} \frac{f^{2}(p)}{p}\right)^{1 / 2}\left(\sum_{[n / 2] \leq p<n} \frac{1}{p}\right)^{1 / 2} \\
& \quad+B_{n}^{1 / 2} \sum_{[n / 2] \leq p<n} \frac{1}{p}=O\left(B_{n}^{1 / 2}\right)=o\left(A_{n}\right)
\end{aligned}
$$

which shows that $A_{n}$ is slowly varying. Assume now $\mathbf{E}|X|<\infty$ and put

$$
L(t)=\#\left\{n: \sum_{k=1}^{n} f(k) \leq t f(n)\right\}
$$

According to Lemmas 2.2 and 2.3, in order to prove Theorem 1.1, it suffices to prove

$$
\mathbf{E} X^{2} \int_{y \geq|X|} \frac{L(y)}{y^{3}} d y<\infty .
$$

To establish (3.2), we use a probabilistic trick employed by Weber in [13. Put

$$
f_{1}(n)=\sum_{d_{0} \leq p \leq n^{1 / 4}} f(p) \chi(p \mid n)
$$

where $d_{0}$ is the same constant as in Lemma 2.4 of [13]. Using $f \geq 0$, (1.12), the slow variation of $A_{n}$ and the asymptotics for $\sum_{p<n} p^{-1}$ used in the proof of Lemma 2.1 we get for sufficiently large $n$

$$
A_{n} \geq \frac{1}{2} A_{2 n} \geq \frac{1}{2} \sum_{n^{1 / 4} \leq p<2 n} \frac{f(p)}{p} \geq \frac{1}{2} \min _{n^{1 / 4} \leq p \leq n} f(p) \sum_{n^{1 / 4} \leq p \leq n} \frac{1}{p} \geq K \max _{n^{1 / 4} \leq p \leq n} f(p)
$$

with some constant $K>0$, and thus we get, using the fact that $n$ has at most 4 different prime factors exceeding $n^{1 / 4}$,

$$
\left|f(n)-f_{1}(n)\right| \leq \sum_{p \leq d_{0}} f(p)+4 \max _{n^{1 / 4} \leq p \leq n} f(p) \ll A_{n} .
$$

(Note that this is the only point in the proof where the smoothness condition (1.12) is used; if instead of the Lindeberg condition (1.7) plus (1.12) we assume 
(1.8), relation (3.3) is obvious in view of (2.2).) Let $(\Omega, \mathcal{A}, \mathbf{P})$ be the probability space on which the sequence $X_{1}, X_{2}, \ldots$ is defined, and consider the product space $(\Omega, \mathcal{A}, \mathbf{P}) \times(\widetilde{\Omega}, \widetilde{\mathcal{A}}, \widetilde{\mathbf{P}})$, where the second space supports a Bernoulli sequence $\left\{\varepsilon_{i}, i \geq 1\right\}$. Let $\widetilde{\mathbf{E}}$ denote expectation in $(\widetilde{\Omega}, \widetilde{\mathcal{A}}, \widetilde{\mathbf{P}})$ and set $S_{n}=\varepsilon_{1}+\ldots+\varepsilon_{n}$, $n=1,2, \ldots$. Then by $(2.1)$

$$
L(t) \leq \#\left\{n: n A_{n} \leq \operatorname{Ctf}(n)\right\} \leq \#\left\{n: S_{n} A_{S_{n}} \leq \operatorname{Ctf}\left(S_{n}\right)\right\},
$$

and this is true for any $t>0$, simply because the graph of the random walk $\left\{S_{n}, n \geq 1\right\}$ replicates all positive integers with possible multiplicities. By the strong law of large numbers $\lim _{n \rightarrow \infty} S_{n} / n \stackrel{a . s .}{=} 1 / 2$, and thus

$$
S_{n} A_{S_{n}} \sim(n / 2) A_{n / 2} \sim(n / 2) A_{n} \quad \text { a.s. }
$$

Here we used the fact that $A_{n}$ is slowly varying and thus by the uniform convergence theorem for slowly varying functions (see e.g. Bingham et al. [1, Theorem 1.2.1]) if $\lambda_{n} \sim \mu_{n}$ are two integer sequences, then $A_{\lambda_{n}} \sim A_{\mu_{n}}$. Thus if $\Omega_{\eta}=\left\{S_{n} A_{S_{n}} \geq\right.$ $\left.\eta n A_{n}, \forall n \geq 1\right\}$, then for some $\eta>0, \mathbf{P}\left(\Omega_{\eta}\right)>0$. Also, reading (3.4) on $\Omega_{\eta}$ gives

$$
L(t) \leq \#\left\{n: n A_{n} \leq(C t / \eta) f\left(S_{n}\right)\right\} \quad \text { on } \Omega_{\eta} \text {, for all } t>0 .
$$

But for all $t>0$

$$
\begin{aligned}
\frac{1}{t} \#\left\{n: n A_{n}\right. & \left.\leq t f\left(S_{n}\right)\right\} \leq 1+\frac{1}{t} \#\left\{n \geq t: n A_{n} \leq t f\left(S_{n}\right)\right\} \\
= & 1+\frac{1}{t} \sum_{n \geq t} \chi\left\{\left(n A_{n}\right)^{2} \leq t^{2} f^{2}\left(S_{n}\right)\right\} \leq 1+t \sum_{n \geq t} \frac{f^{2}\left(S_{n}\right)}{\left(n A_{n}\right)^{2}} .
\end{aligned}
$$

Further, by Lemma 2.4 there exists a constant $C^{*}$ such that

$$
\begin{aligned}
\widetilde{\mathbf{E}} f_{1}^{2}\left(S_{n}\right) & =\widetilde{\mathbf{E}}\left\{\sum_{d_{0} \leq p \leq\left(S_{n}\right)^{1 / 4}} f(p) \chi\left(p \mid S_{n}\right)\right\}^{2} \leq \widetilde{\mathbf{E}}\left\{\sum_{d_{0} \leq p \leq n^{1 / 4}} f(p) \chi\left(p \mid S_{n}\right)\right\}^{2} \\
& =\sum_{d_{0} \leq p_{i_{1}}, p_{i_{2}} \leq n^{1 / 4}} f\left(p_{i_{1}}\right) f\left(p_{i_{2}}\right) \widetilde{\mathbf{E}} \chi\left(p_{i_{1}} p_{i_{2}} \mid S_{n}\right) \\
& \leq C^{*}\left\{\sum_{p_{i_{1}}, p_{i_{2}} \leq n^{1 / 4}} \frac{f\left(p_{i_{1}}\right) f\left(p_{i_{2}}\right)}{p_{i_{1}} p_{i_{2}}}\right\} \leq C^{*}\left\{\sum_{p \leq n^{1 / 4}} \frac{f(p)}{p}\right\}^{2} \leq C^{*} A_{n}^{2},
\end{aligned}
$$

provided $n$ is sufficiently large, which from now on we assume. From (3.3), (3.7) and Minkowski's inequality it follows that

$$
\widetilde{\mathbf{E}} f^{2}\left(S_{n}\right) \leq C^{\prime} A_{n}^{2} .
$$

Therefore

$$
\widetilde{\mathbf{E}} \frac{1}{t} \#\left\{n: n A_{n} \leq t f\left(S_{n}\right)\right\} \leq 1+t \sum_{n \geq t} \frac{\widetilde{\mathbf{E}} f^{2}\left(S_{n}\right)}{\left(n A_{n}\right)^{2}} \leq 1+C^{\prime} t \sum_{n \geq t} \frac{1}{n^{2}} \leq C^{\prime \prime}<\infty .
$$

It follows that

$$
\mathbf{E} \widetilde{\mathbf{E}} X^{2} \int_{y \geq|X|} \frac{\#\left\{n: n A_{n} \leq y f\left(S_{n}\right)\right\}}{y^{3}} d y \leq C^{\prime \prime} \mathbf{E} X^{2} \int_{y \geq|X|} \frac{1}{y^{2}} d y \leq C^{\prime \prime} \mathbf{E}|X|<\infty .
$$


Also, in view of (3.5) and Fubini's Theorem

$$
\begin{aligned}
& \widetilde{\mathbf{E}} \chi\left(\Omega_{\eta}\right) \cdot \mathbf{E} X^{2} \int_{y \geq|X|} \frac{L(y)}{y^{3}} d y \leq \mathbf{E} \widetilde{\mathbf{E}} X^{2} \int_{y \geq|X|} \frac{\#\left\{n: n A_{n} \leq(C y / \eta) f\left(S_{n}\right)\right\}}{y^{3}} d y \\
& \quad \leq C^{\prime \prime \prime} \mathbf{E}|X|<\infty
\end{aligned}
$$

As

$$
\widetilde{\mathbf{E}} \chi\left(\Omega_{\eta}\right) \cdot \mathbf{E} X^{2} \int_{y \geq|X|} \frac{L(y)}{y^{3}} d y=\mathbf{P}\left\{\chi\left(\Omega_{\eta}\right)\right\} \mathbf{E} X^{2} \int_{y \geq|X|} \frac{L(y)}{y^{3}} d y,
$$

condition (3.2) is obtained and Theorem 1.1 is thus proved.

Remark. It is quite interesting to observe by the randomization argument we used in the above proof that the term " $\frac{1}{p}$ " in the summand of $A_{n}$ appears in (3.7) as the expectation of a random factor.

Proof of Theorem 1.2. In view of Lemma 2.5 and the equivalence of (2.6) and (2.8), it suffices to verify that

$$
\mathbf{E} X^{4} \int_{y \geq X^{2}} \frac{\Lambda(y)}{y^{3}} d y<\infty
$$

where

$$
\Lambda(y)=\#\left\{n: \sum_{k=1}^{n} f^{2}(k) \leq y f^{2}(n)\right\} .
$$

The proof being very similar, we only mention the modifications. We replace $f_{1}(n)$ by

$$
f_{2}(n)=\sum_{d_{0} \leq p \leq n^{1 / 8}} f(p) \chi(p \mid n),
$$

where $d_{0}$ is the same constant as in Lemma 2.4. Similarly to (3.3), we get

$$
\left|f(n)-f_{2}(n)\right| \ll A_{n} .
$$

By using the same randomization argument as above and applying (2.1), we see that the set $\Omega_{\eta}^{*}$ defined by

$$
\Omega_{\eta}^{*}=\left\{S_{n} A_{S_{n}}^{2} \geq \eta n A_{n}^{2}, \forall n \geq 1\right\}
$$

has positive probability for some $\eta>0$, and on $\Omega_{\eta}^{*}$ we have for all $y>0$

$$
\Lambda(y) \leq \#\left\{n: n A_{n}^{2} \leq\left(C_{0} y / \eta\right) f^{2}\left(S_{n}\right)\right\}
$$

for some positive constant $C_{0}$. Instead of (3.6) we have

$$
\begin{aligned}
\frac{1}{t} \#\left\{n: n A_{n}^{2} \leq t f^{2}\left(S_{n}\right)\right\} & \leq 1+\frac{1}{t} \#\left\{n \geq t: n A_{n}^{2} \leq t f^{2}\left(S_{n}\right)\right\} \\
& =1+\frac{1}{t} \sum_{n \geq t} \chi\left\{n^{2} A_{n}^{4} \leq t^{2} f^{4}\left(S_{n}\right)\right\} \leq 1+t \sum_{n \geq t} \frac{f^{4}\left(S_{n}\right)}{n^{2} A_{n}^{4}} .
\end{aligned}
$$


Now,

$$
\begin{aligned}
\widetilde{\mathbf{E}} f_{2}^{4}\left(S_{n}\right) & =\widetilde{\mathbf{E}}\left\{\sum_{d_{0} \leq p \leq\left(S_{n}\right)^{1 / 8}} f(p) \chi\left(p \mid S_{n}\right)\right\}^{4} \leq \widetilde{\mathbf{E}}\left\{\sum_{d_{0} \leq p \leq n^{1 / 8}} f(p) \chi\left(p \mid S_{n}\right)\right\}^{4} \\
& =\sum_{d_{0} \leq p_{i_{1}}, p_{i_{2}}, p_{i_{3}}, p_{i_{4}} \leq n^{1 / 8}} f\left(p_{i_{1}}\right) f\left(p_{i_{2}}\right) f\left(p_{i_{3}}\right) f\left(p_{i_{4}}\right) \widetilde{\mathbf{E}} \chi\left(p_{i_{1}} p_{i_{2}} p_{i_{3}} p_{i_{4}} \mid S_{n}\right) \\
& \leq C^{*}\left\{\sum_{p_{i_{1}}, p_{i_{2}}, p_{i_{3}}, p_{i_{4}} \leq n^{1 / 8}} \frac{f\left(p_{i_{1}}\right) f\left(p_{i_{2}}\right) f\left(p_{i_{3}}\right) f\left(p_{i_{4}}\right)}{p_{i_{1}} p_{i_{2}} p_{i_{3}} p_{i_{4}}}\right\} \leq C^{*}\left\{\sum_{p \leq n^{1 / 8}} \frac{f(p)}{p}\right\}^{4} \\
& \leq C^{*} A_{n}^{4},
\end{aligned}
$$

provided $n$ is sufficiently large, which from now on we assume. From (3.10) and (3.11) it follows that

$$
\widetilde{\mathbf{E}} f^{4}\left(S_{n}\right) \leq C^{\prime} A_{n}^{4}
$$

Thus instead of (3.9) we get

$$
\widetilde{\mathbf{E}} \frac{1}{t} \#\left\{n: n A_{n}^{2} \leq t f^{2}\left(S_{n}\right)\right\} \leq 1+t \sum_{n \geq t} \frac{\widetilde{\mathbf{E}} f^{4}\left(S_{n}\right)}{n^{2} A_{n}^{4}} \leq 1+C^{\prime} t \sum_{n \geq t} \frac{1}{n^{2}} \leq C^{\prime \prime}<\infty .
$$

To conclude, we now operate exactly as at the end of the previous proof.

Remark. The reduction argument based on (3.3) we used to treat the case of additive arithmetical functions is no longer valid when passing to multiplicative functions, e.g. the usual divisor function $d$. However, the argument applies to the truncated divisor function $d_{1}(n)=\sum_{d \leq n^{1 / 4}} \chi(d \mid n)$, and gives for any sequence $\mathcal{X}$ of centered, independent, identically distributed, integrable random variables

$$
\lim _{N \rightarrow \infty} \frac{\sum_{n=1}^{N} d_{1}(n) X_{n}}{\sum_{n=1}^{N} d_{1}(n)} \stackrel{a . s .}{=} \mathbf{E} X
$$

A similar result can be obtained for the LIL with the truncated divisor function $d_{2}(n)=\sum_{d \leq n^{1 / 8}} \chi(d \mid n)$. We omit the details of proofs, which are quite similar to the above.

\section{REFERENCES}

[1] Bingham N., Goldie, C. and Teugels, J., [1987] Regular Variation, Cambridge University Press. MR0898871 (88i:26004)

[2] Elliott, P. D., [1980] Probabilistic number theory II, Springer, New York. MR0560507 (82h:10002b)

[3] Erdős, P., Kac, M., [1940] The Gaussian law of errors in the theory of additive numbertheoretic functions, Amer. J. Math. 62, 738-742. MR0002374 (2:42c)

[4] Fisher E., [1992] A Skorohod representation and an invariance principle for sums of weighted i.i.d. random variables, Rocky Mount. J. Math. 22, 169-179. MR.1159950 (93e:60065)

[5] Halberstam H., [1955] On the distribution of additive number-theoretic functions I, J. London Math. Soc. 30, 43-53. MR0066406 (16:569g)

[6] Jamison B., Orey S., Pruitt W., [1965] Convergence of weighted averages of independent random variables, Z. Wahrscheinlichkeitsth. 4, 40-44. MR0182044 (31:6268)

[7] Kubilius, J., [1964] Probabilistic Methods in the Theory of Numbers, Amer. Math. Soc. Translations of Math. Monographs, 11 Providence. MR0160745 (28:3956)

[8] Philipp, W., [1971] Mixing sequences of random variables and probabilistic number theory, Memoirs of the AMS, No. 117. MR0437481 (55:10411) 
[9] Shapiro, H. N., [1956] Distribution functions of additive arithmetic functions, Proc. Nat. Acad. Sci. USA 42, 426-430. MR0079609 (18:113c)

[10] Weber M., [2005] An ergodic theorem of arithmetical type, Tatra Mountains Math. J. 31, 123-129. MR2208793

[11] Weber M., [2004] An LIL of arithmetical type, preprint.

[12] Weber M., [2004] An arithmetical property of Rademacher sums. Indagationes Math. 15, 133-150. MR2061474 (2005d:60051)

[13] Weber M., [2006] On the order of magnitude of the divisor function, Acta Math. Sinica 22, 377-382. MR2214359

[14] Weber M., [2004] Divisors, spin sums and the functional equation of the Zeta-Riemann function. Periodica Math. Hungar. 51, 1-13. MR2180637

Institut für Statistik, Technische Universität Graz, Steyrergasse 17/IV, A-8010 Graz, Austria

E-mail address: berkes@tugraz.at

Mathématique (IRMA), Université Louis-Pasteur et C.N.R.S., 7 Rue René Descartes, 67084 Strasbourg Cedex, France

E-mail address: weber@math.u-strasbg.fr 